This letter was shown to Dr Fitch who replies as follows:

SiR,

The proband described by Goswami and Chaurasia was thought to have brachydactyly type $B$ because of the appearance of the digits in $x$-rays of the hands and feet. However, for any given family one can only have an opinion as to whether that particular syndrome fits into a given spectrum of anomalies. There are at least three features in the proband which are indeed different from the usual brachydactyly B subjects. The fingers seem to taper more at the distal ends, the toe syndactyly is much more severe, and digits 2 and 3 on the left hand are not less severely affected than digits 4 and 5 . However, the right hand, which appears to be normal, may have a very minor expression of the gene as the distal phalanx of digit 4 and perhaps digit 3 seems to be reduced in length (the distal phalanx of digit 5 is not shown in fig 3). If this is true, then the typical pattern of digits 4 and 5 being more affected would be present in this hand. Rudimentary or even normal nails are found quite frequently, so that this cannot be used as an argument against the diagnosis of brachydactyly B. Many other family members have this trait and a report of other affected subjects would be very useful in determining whether or not this family belongs in the spectrum of brachydactyly B.

N Frrch

Lady Davis Institute for Medical Research, Sir Mortimer B Davis Jewish General Hospital, 3755 Cote Saint Catherine Road, Montreal, Quebec, Canada H3T 1E2

\section{Autosomal dominant inheritance of Gerhardt's syndrome in three generations of a family}

SIR,

Bilateral paralysis of the abductor laryngeal muscles (Gerhardt's syndrome) may be secondary to bulbar lesions of various kinds (inflammatory, ischaemic, degenerative). The syndrome is well known to laryngologists because it entails a permanent state of inspiratory dyspnoea which may require surgery.

Plott $^{1}$ has described a familial case of this syndrome in three sibs born to healthy, non-consanguineous parents. They all also had mental retardation and in one there was an inner ear deafness. A fourth sib had died from asphyxia a few hours after birth. The likelihood of an X linked recessive gene was estimated by the author as 1 in

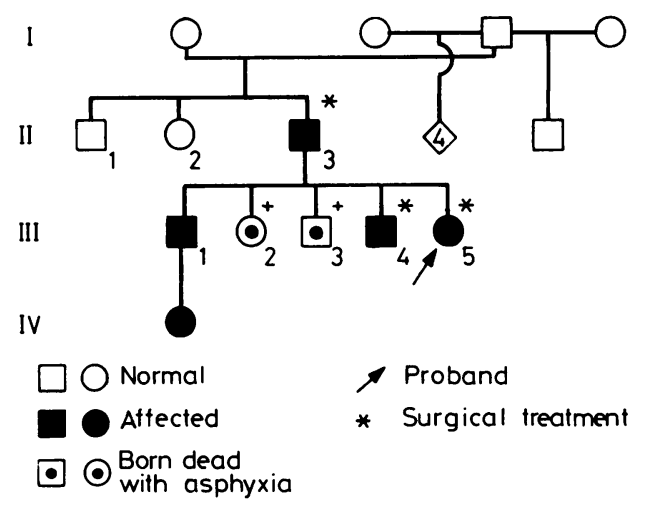

FIGURE Family pedigree.

16, of an autosomal dominant gene as 1 in 256, and of an autosomal recessive gene as 1 in 4096.

We have observed a family with paralysis of the abductors in three generations (fig). All affected subjects had normal mental development. Neurological and audiometric examination was noncontributory and the electroencephalogram was normal. The severity of the respiratory disorder was variable in the subjects. Two died of neonatal asphyxia, one has partial paralysis with mild symptoms, and in three subjects it has been necessary to carry out an aritenoidopexia at different ages. This observation differs from those reported by Plott $^{1}$ because of the absence of mental retardation. In addition, in our family the inheritance pattern was clearly autosomal dominant. Therefore, we favour the view that this is a distinct genetic entity.

\section{G Morelli, ${ }^{*}$ C Mesolella, ${ }^{*}$ M l Cavaliere, $\dagger$ M Stabile, $\dagger$ and V Ventruto $\dagger$ *Clinica Otorinolaringoiatrica I Facoltà di Medicina dell'Università, and †Servizio Genetica Medica, Ospedale Cardarelli, Napoli, Italy}

\section{Reference}

1 Plott D. Congenital laryngeal-abductor paralysis due to nucleus ambiguus dysgenesis in three brothers. $N$ Engl J Med 1964;271:593-7.

\section{Tuberous sclerosis}

SIR,

Tuberous sclerosis is an autosomal dominant disorder with variable expression that includes typical cutaneous lesions, hamartomas of the brain, retina, kidney, and lung, sclerotic or cystic skeletal 